\title{
S2 Table
}

Primer used to detect the deletions generated by CRISPR/Cas9

\begin{tabular}{|l|l|l|}
\hline Primer to detect deletions in R1 & Target region & Position (hg19) \\
\hline P2_F & TGTGGCTTGGCCTAATTG & \multirow{2}{*}{ chr5:36743828-36749685 } \\
P2_R & CAGAGGCTGGTTCTCTTG & \\
\hline \hline P3_F & AACGCGAGCCTCTTCTCTTCC & \multirow{2}{*}{ chr5:36737654-36749678 } \\
P3_R & TGGTTCTCTTGGTCCAGGAGG & \\
\hline \hline P4_F & CCCAACACCTGTTCCTCTTAC & \multirow{2}{*}{ chr5:36737678-36737840 } \\
P4_R & CCTGGGACTGACATTTCTGTTC & \\
\hline \hline P5_F & CTCAAGGGCAGACACAATG & \multirow{2}{*}{ chr5:36742317-36742621 } \\
P5_R & GGCACTTACTCTGTGAAAGG & \\
\hline \hline P6_F & GCCCAAATAGCACTGCAGAC & \multirow{2}{*}{ chr5:36743671-36744015 } \\
P6_R & GAAAGTGGCAGAGCTGTGAG & \\
\hline \hline P7_F & GGTACATGGCTCCCATTTGAAC & \multirow{2}{*}{ chr5:36746584-36746885 } \\
P7_R & AGTGCCTAGGTAAGGAACCTTG & \\
\hline \hline P8_F & ACTACATGCCAGGCACAATC & \multirow{2}{*}{ chr5:36748530-36748959 } \\
P8_R & TGGCATTTCTGGCCTAACTC & \\
\hline
\end{tabular}

